\title{
Medical Application of Vest Pocket Computers
}

\author{
Ralph Grams, M.D.
}

\begin{abstract}
This special editorial review in the Journal of Medical Systems is dedicated to the use and application of vest pocket computers (VPCs). We will not be discussing PCs, mainframes, lap tops, or notebook-size processors. Our focus is on a light-weight, small, compact device that will comfortably fit in the vest pocket or purse of a health care worker. This new device, the VPC, is just now coming into the market with major manufacturer support. This issue will review the current specifications for such equipment and also available application software that can be purchased. The topics discussed in this review will follow a logical sequence of presentation. First, will be a justification of the VPC in light of the needs of the user community. The second section will discuss the available hardware on the market and present a direct comparison of prices and specifications. Our final overview will offer a projection for the future and a discussion of where this technology might lead in the coming months. Today, we are at the threshold of a major innovation in the practice of medicine. The VPC will be a serious step forward in the delivery of knowledge to a wide variety of users who are limited in their current application of computer technology. I hope that this introductory series will be the stimulus for a user group to develop and initiate a flood of activity that will generate great interest in this new technology.
\end{abstract}

\section{A JUSTIFICATION FOR VEST POCKET COMPUTING}

\section{What Is a Vest Pocket Computer?}

Before we launch into an extensive justification for another tool in the practice of medicine, we must first agree upon some common terms, definitions, and characteristics.

1. A vest pocket computer (VPC) is a stand-alone digital computer that will comfortably fit in the vest pocket of a suit coat. The size is limited to approximately 6 in. by $4 \mathrm{in}$. by $1 \mathrm{in}$. and the weight must not be more than $1 \mathrm{lb}$.

From the Medical Systems Group, College of Medicine, University of Florida, Gainesville, Florida 32610. 
2. The VPC must not be a burden to carry or to use.

3. The device must have a display screen, keyboard, and mass data storage.

4. There should be sufficient flexibility in the VPC so that new applications and databases can be added when needed.

5. The device must have sufficient power for extended remote use (2-3 months of routine use).

6. The VPC should have provision for charging or replacing the batteries without destroying the files and storage media.

7. The keyboard should be flexible and provide for alpha as well as numeric data entry.

8. Special keys should be present that will expedite the handling of the screen.

9. The device should permit the uploading and downloading of data from a PC.

10. The package should be rugged and able to survive frequent use and occasional abuse.

11. Service should be readily available and the retail price for all this capability should not exceed $\$ 300.00$ !

Can such a computer be built and will it ever exist? The answer is an obvious "Yes". We now have several devices on the market that will qualify for the VPC label and there are new versions being introduced almost every month.

\section{Why Do We Need a Vest Pocket Computer?}

Computers have been in medical practice since their early introduction in the 1960s. Mainframes dominated this early period and only the brave would attempt anything besides a financial system. Over these last 25-30 years, every effort has been made to incorporate computers into the mainstream of medicine. In a recent report of the AMA, we find that in 1990 , less than $50 \%$ of all doctors use any type of computer in their practice. ${ }^{1}$ Eighty-five percent of those using a computer use it for financial processing of their patient accounts. In most cases, these computers are operated by staff who insulate the physician from any hands-on knowledge of the system or its inner workings.

The jury is "in" on the use of computers in health care and the verdict is obvious. For the most part, the practice of medicine has survived without being infected by the computer virus. The physician is still king of his domain and does not need a computer to practice medicine.

With this basic resistance to change and demonstrated lack of interest, the average health care provider has not benefited from this technical revolution and still awaits awakening.

\section{There Are Several Reasons for This General Lack of Interest}

First, medicine is very complex and each case is unique. Software applications have not recognized this fact and have tended to make the practice of medicine like a cookbook where one solution fits all. This may make good marketing sense when selling hats and pantyhose, but will not impress a physician who only sees exceptions to the "rule."

Too often, the programs that have been developed are designed to replace rather than extend the potential of a physician. Almost every approach to diagnostic systems has been 
attempted without enduring success. All of them are incomplete and lack the sophistication of an average physician. Therefore, they have not found commercial success in the market, and do not represent a significant improvement in the delivery of health care. Many of these programs have been widely advertised as superhuman. When they have been tested with real cases and complex environments, there are few who will stand up in their defense. Many of these programs represent the ideas of the author and are not accepted in the general medical community. They have a biased viewpoint and have not had sufficient peer review and independent testing to really prove their viability.

Another reason for the computers lack of success in the medical market is the physical equipment itself. To use a standard computer you must sit down and interact with the device on a keyboard and screen. For most physicians, this is not their normal routine. Physicians and healthcare providers are extremely active and mobile. Their day does not revolve around a computer terminal, but a series of patients who will be located in many parts of the city in which they practice. They use the telephone frequently and will be paged and moved around their territory in a random fashion. There are some basic rules in designing medical systems that interact with doctors.

1. Physicians will not wait in line to use a terminal.

2. Physicians will not tolerate more than a 3- to 4-sec delay in screen response.

3. Physicians will probably not use a system that fails to produce an answer to their question in three to five screen interactions.

Anyone who has installed a hospital computer system will recognize these unwritten rules which must not be violated. ${ }^{2}$

Physicians as a group are rather practical people. When they are presented with a new tool or technology, they will immediately ask themselves if this will make their work easier or more efficient. If the answer to either of these questions is "Maybe?" or "No!," then the tool or technology will most likely be rejected.

A practical demonstration of this principle is evident today. Over the past 5 years, the use of computers in radiology has mushroomed. Why? In the simplest terms, the computer can do something that the human brain and the physician can not do. The ability to do three-dimensional representations of two-dimensional pictures is a real step forward. The ability to abstract clinically useful data from a magnetic field is totally outside the domain of the physician. Physicians are now addicted to this new technology, because it performs a useful function and can not be duplicated by the human brain.

Based on a proven lack of success and 25 years of effort, a new vision is needed for healthcare computing that takes into account the limitations of the end user, the physician, and builds upon the principles of good scholarship. We know what has not worked, and we should be able to project a course of action that will make a product that will work.

It is the author's belief that four factors must coexist before there will be a serious change in the general attitude of most physicians:

1. The application packages must support good medical practice at the highest academic level.

2. The software must not be designed to compete with the physician.

3. The hardware must be of vest pocket size and must provide full power on an instantaneous basis.

4. The hardware and software must be inexpensive and compete with other current media such as journals and books. 
In this time of rapid technologic change, one of the greatest needs physicians have is to stay current with the fast pace of medical knowledge. Over 75,000 new articles are being published in medicine each year and the list of new journals increases daily.

The human brain was never designed to function as a mass storage device and fails miserably in this effort with age. Here, I believe, is the basis for a real contribution to medicine. There is a specific need for tools that extend the human memory and keep pace with the rapid changes in the field. This need extends from medical school to the day a doctor or health care workers retires from practice.

Although massive databases have been accumulated, there has been no vehicle for general distribution that is accepted by the healthcare community. Mainframes and PCs have not done the job, and we still have an unmet need that remains a challenge to this very day.

I believe the greatest barrier to the general use of computers in medicine is the convenience factor. Is the unit there when I need it? Can I depend on it when there is an emergency? These are important and legitimate questions. A physician can not place his confidence in something that is not reliable. A patient's life can not depend upon something that is remote or difficult to use. All of these factors lead to one obvious conclusion: "Until the computer is made useful and acceptable to the end user, there will be no acceptance of the technology."

\section{What Would Make a Computer Attractive to a Busy Healthcare Specialist?}

1. Application programs that work and do something useful that the physician can not do himself.

2. Prepackaged software that is simple to use and requires no training.

3. Software that produces useful results in a few key strokes.

4. No dependence on typing.

5. Hardware that is truly portable and that can fit in the vest pocket of a suit coat or in a purse.

6. Hardware that is reliable and rugged enough for daily use.

7. Service and swap facilities on the local basis so that there is no appreciable downtime with the unit.

8. A price for the entire system that is competitive with other sources of information currently available to the physician (a total package price for hardware and software less than $\$ 500.00$ ).

9. A screen that is large enough for several lines of text to be displayed at a time. A graphics feature would be useful to show statistics and small line drawings.

10. A keyboard that allows simple one button control of the screen and menus. The keys might be arrayed like a typewriter or in simple alphabetical order with a numeric key pad. The option of choosing one design or the other would be left to the customer.

11. A simple and inexpensive means of data/program storage is needed. Cards, disks or some form of magnetic media must be employed by the unit to deliver the application software and database.

12. A communication port is highly desirable so that uploading and downloading of data and programs can occur between the physician's PC and the VPC.

13. General software applications that can provide business related tools such as a memo pad, phone number registry, calendar, scheduler, etc. 
14. The backing of a stable computer manufacturer to insure product continuity and future system enhancements.

As strange as it might seem, the production of the hardware is not the difficult part of this wish list. The creation of quality software and databases will be the frontier where the real action must take place. Computer companies know how to miniaturize equipment and make it more powerful. They know absolutely nothing about the practice of medicine and what would be attractive to a physician.

Working with these small machines is a new world for those who are accustomed to 40-80 megabytes of hard disk space. We currently have available 64K ROM cards that can be used for program and data storage. There is a strong possibility that this will rapidly expand to $128-256 \mathrm{~K}$ of space, but beyond this point there is no clear path to the inexpensive megabytes that are common on todays PCs.

This means that databases must be stripped to their essentials and a good deal of preprocessing done to minimize their space. Custom software is needed for each card or module, and there must be a developer who is willing to underwrite the cost of this process. In most cases, this will not be the hardware manufacturer.

In our current environment, we do not have a supply of well tested databases that would be acceptable to the average physician. This will take time to develop and each must be integrated with the VPC for rapid query and display. A production and distribution system does not exist at this time, and we do not have an established market in which to submit new products. In other words, this is very new and very speculative.

\section{Why a VPC and Not Something Else?}

Everything else has been tried. Lap tops are too big to haul around. Portables are too bulky and inconvenient for physician use. PCs are not portable or convenient to use even in the office. The only smaller size that would be acceptable is a wristwatch computer, and that is currently impossible to use, since the display screen would be too small. Price, packaging and performance all lead to the VPC as the natural vehicle for physician use.

Only time and specific test packages will make a final determination as to whether this analysis is correct.

\section{VEST POCKET COMPUTER HARDWARE REVIEW}

\section{Overview}

The age of pocket computing is now here. The stage is set for a market showdown and a great period of growth and product development. As of the writing of this article, there are five major players in the vest pocket computer market: Atari, Casio, Psion, Sharp, and Selectronics. This paper will present the basic system specifications of each and show what differences exist between the available hardware on the market. It is anticipated that this review will be out of date before it is ever printed, since this field is expanding with new product releases almost every month.

\section{Background}

Desk-top computers, portables, lap tops, and notebook-sized computer packages have all been introduced into the commercial market. So far, the general practice of 
medicine has found none of these units terribly attractive. For the most part, they are too big, too expensive, too heavy, and too inconvenient to use. Until the computer becomes as small and lightweight as a pager or wallet, there will be little chance of finding general acceptance of the computer as a partner in clinical medicine.

With the recent release of VPCs, a new potential exists to reach a group of individuals who have found no hardware package attractive to their lifestyle. Physicians, in general, will not type or slow down for general clerical work. They also will not learn to program the computer. Any attempt at selling them this type of production package will not work and there are many companies that can validate this conclusion.

The VPC is different:

1. The price is under $\$ 300.00$.

2. The package can go anywhere in vest pocket or purse.

3. The unit is self contained and light weight.

4. The hardware offers the option of accessing databases that can be custom designed for the user.

5. The unit is fun to use and will be a status symbol once the software packages take hold in the market.

\section{Hardware Comparison}

Tables 1-5 list the available products that would truly qualify as vest pocket computers. There are a wide variety of packages and many options that make each unit unique.

Although the hardware is in place, there is only one commercial medical package for sale as of this writing and that is a medical speller. Medical Economics Inc. has indicated that they are producing a VPC PDR which will be available in the latter part of 1990 . Professional Computerized Notebooks Inc. of Englewood, N.J. (201-816-0620), has demonstrated a drug interaction card for the Sharp Wizard computer. No other commercial options have yet appeared in the market.

This is further verification of the general principle that will apply in this field. Namely, that the hardware will always be ahead of the software and databases. As more developers come into the industry, this lag in production will decrease and there should be a good selection of products in the next 2-4 years.

Since there is no basic medical software to evaluate at this time, our focus will be primarily on the hardware. In looking at the commercially produced units, there are several obvious areas of comparison.

1. Case size and weight. No one wants to look like a detective packing a revolver in his coat. The unit must be light weight and slim to make the package acceptable to the medical community.

2. The screen must be readable and have sufficient character display space to handle medical text.

3. The keyboard must offer limited typing capability. A QWERTY (typewriter layout) design is preferred.

4. The unit must offer mass storage capability to handle medical databases.

5. The VPC package must allow several applications to be carried in the package for a broad range of use. 
Table $1^{a}$

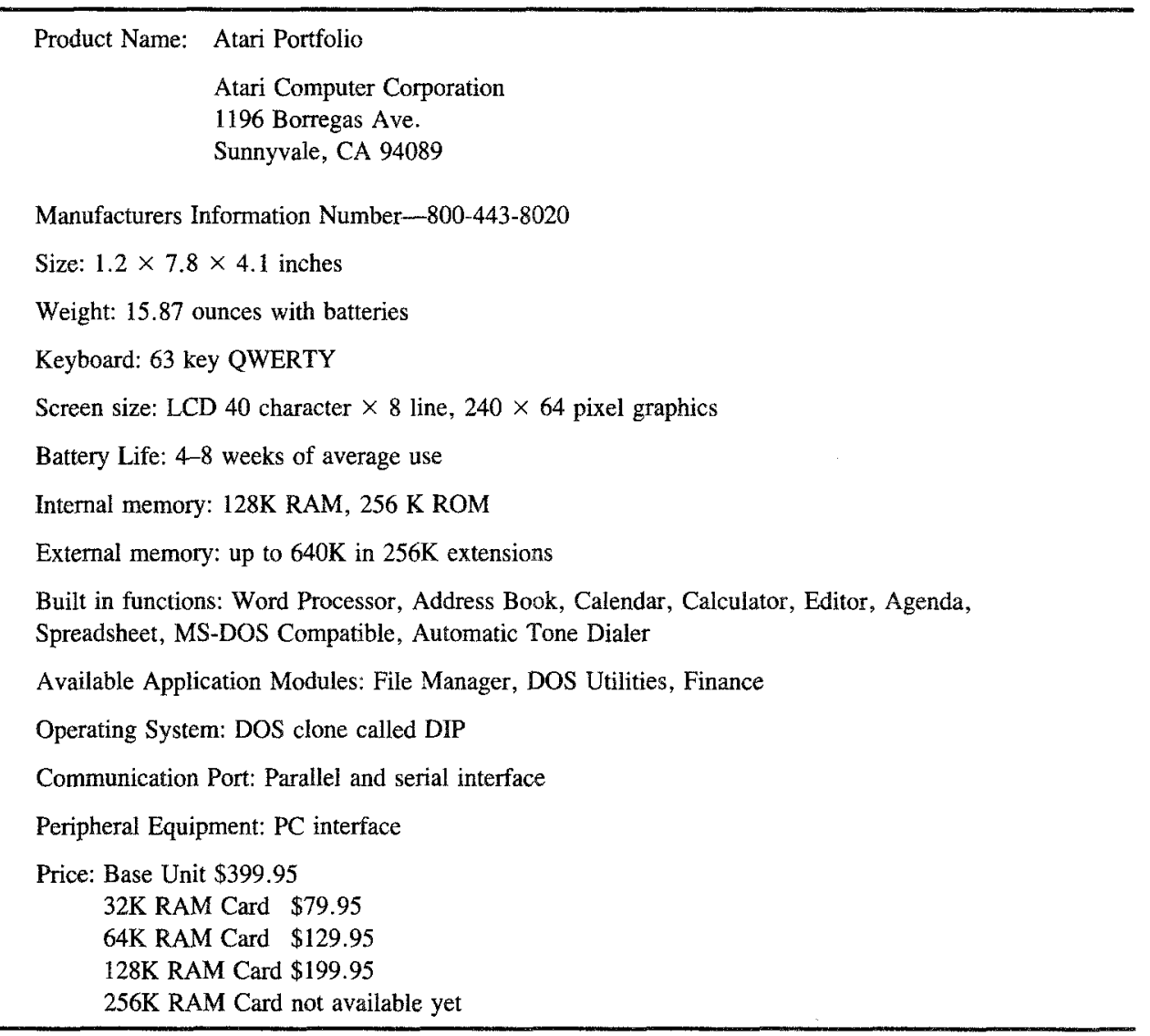

${ }^{a}$ See Figure 1.

Besides the computer, there are several peripheral options that would be highly desirable. The unit should allow for an upload and a download from a standard PC. This could provide a direct interface to the office computer and permit a method of transferring daily records and billing information.

Another possible peripheral would be a small printer that could allow a printed copy of any record or transaction. A modem might be useful in dealing with telephone transactions.

\section{What Could Be Expected in the Future?}

1. Larger ROM cards or storage modules and more processing power will be available in a small light-weight package.

2. A larger screen is planned by most manufacturers with more lines of display. Perhaps a color display will be possible for highlighting and graphics presentation.

3. In the future, we may see the integration of the VPC with the pager which is so common on most doctors and nurses. 


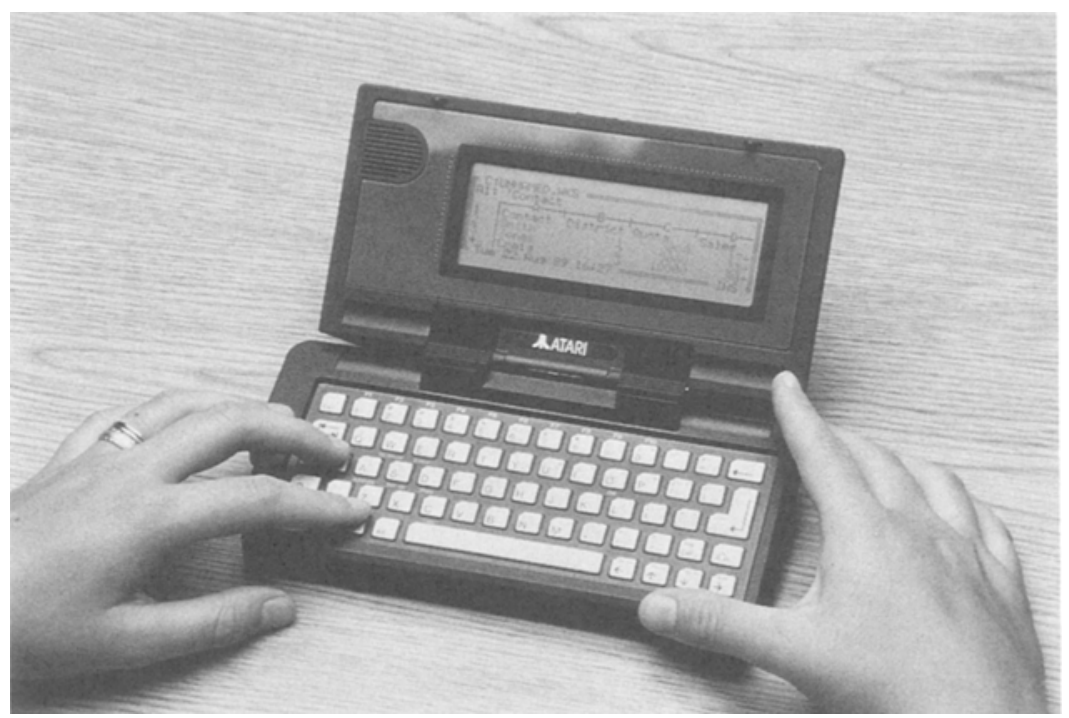

Figure 1. The Atari Portfolio is a handheld MS-DOS compatible computer. In a split case design, the screen is 40 characters by 8 lines on an LCD, and the QWERTY keyboard offers a typing option for user interaction. The computer is an Intel $80 \mathrm{C} 88$ running at $4.9 \mathrm{MHz}$ with an MS-DOS 2.11 operating system.

4. Integration of the VPC, pager, and the cellular telephone are possible in one slim pocket package. Now that would be one terrific product if the price were realistic. One would then carry the VPCTP-Vest pocket computer, telephone, and pager.

\section{What Are the Growth Limiting Factors for the VPC?}

1. Functional software that is desired by the medical community.

2. Organizational support for a new product and services from a major medical sponsor.

3. Sufficient mass storage capacity to house the large medical databases that exist in the field. basis.

4. A price that is attractive with service and product assistance on a 24 hour a day

5. Frequent updates to the databases so that files are current and accurate (annual release of new ROM cards or modules).

6. Integration of ROM cards and major textbooks so that abstracted versions of the texts could be available on the VPC. This could be packaged with the book or sold separately.

7. Diagnostic packages with sufficient documentation could be miniaturized and distributed via VPC technology.

\section{Overview of Market Direction}

In its initial development stage, there appears to be a tradeoff between function and features. As you review Tables 1-5 it is evident that most of the units classified as a VPC 
Table $2^{a}$

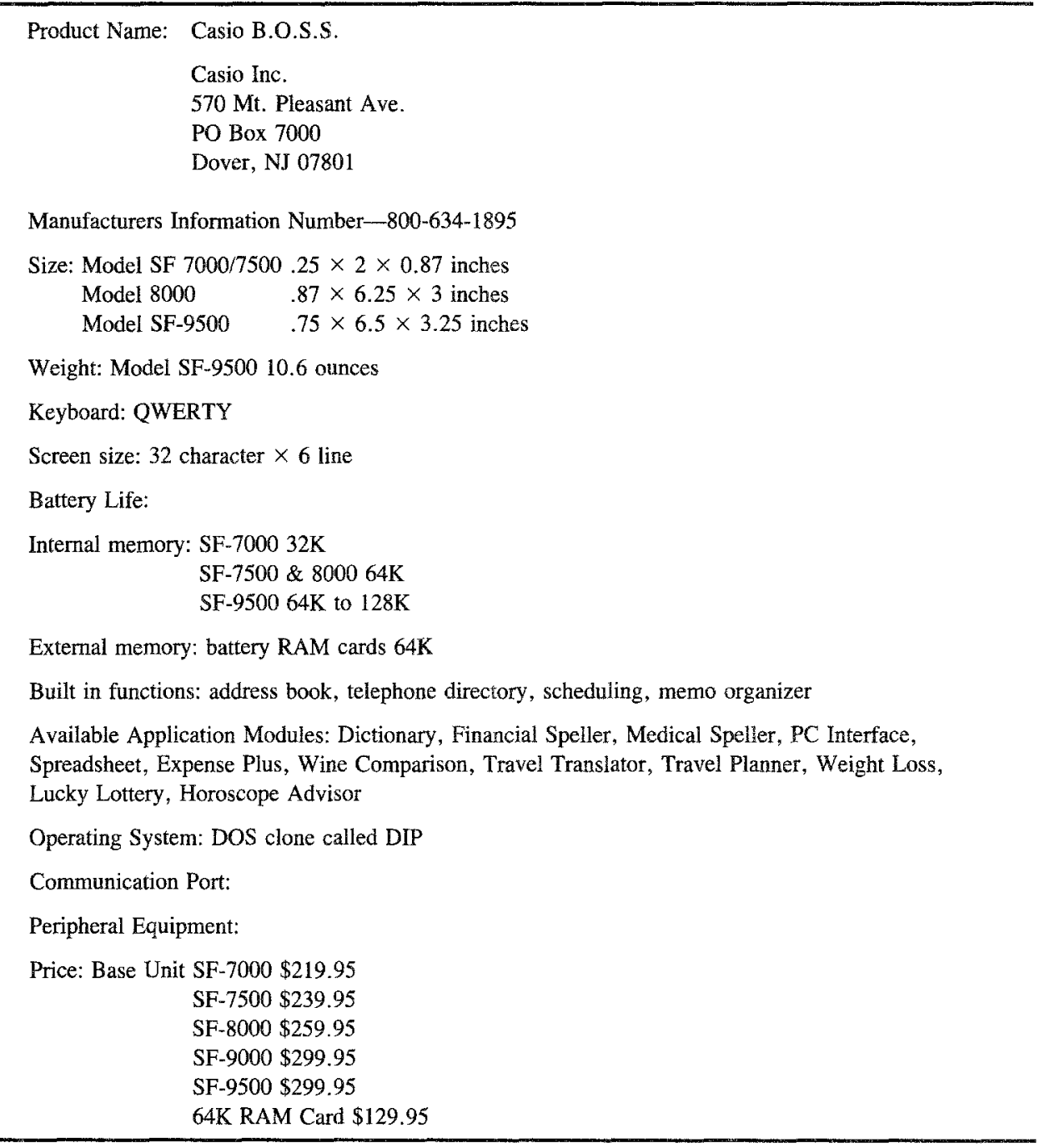

\footnotetext{
${ }^{a}$ See Figure 2.
}

are really nothing more than a computer version of a pocket secretary. I believe that this was the original design and continues as the major market thrust by the computer manufacturers. The idea of a flexible attached database has grown from the original business design and is really secondary to the fixed business packages that are being developed.

With most of the VPC hardware packages as they stand, there is insufficient space for medical databases and a lack of display capability for text. The Selectronics hardware is an exception to this trend, since it can handle large databases, but is not designed as a multipurpose unit with business interface capabilities. The market will determine how quickly these two design strategies can merge and will set the stage for competition equivalent to the PC and its many clones. Although it would be ideal to contemplate a 


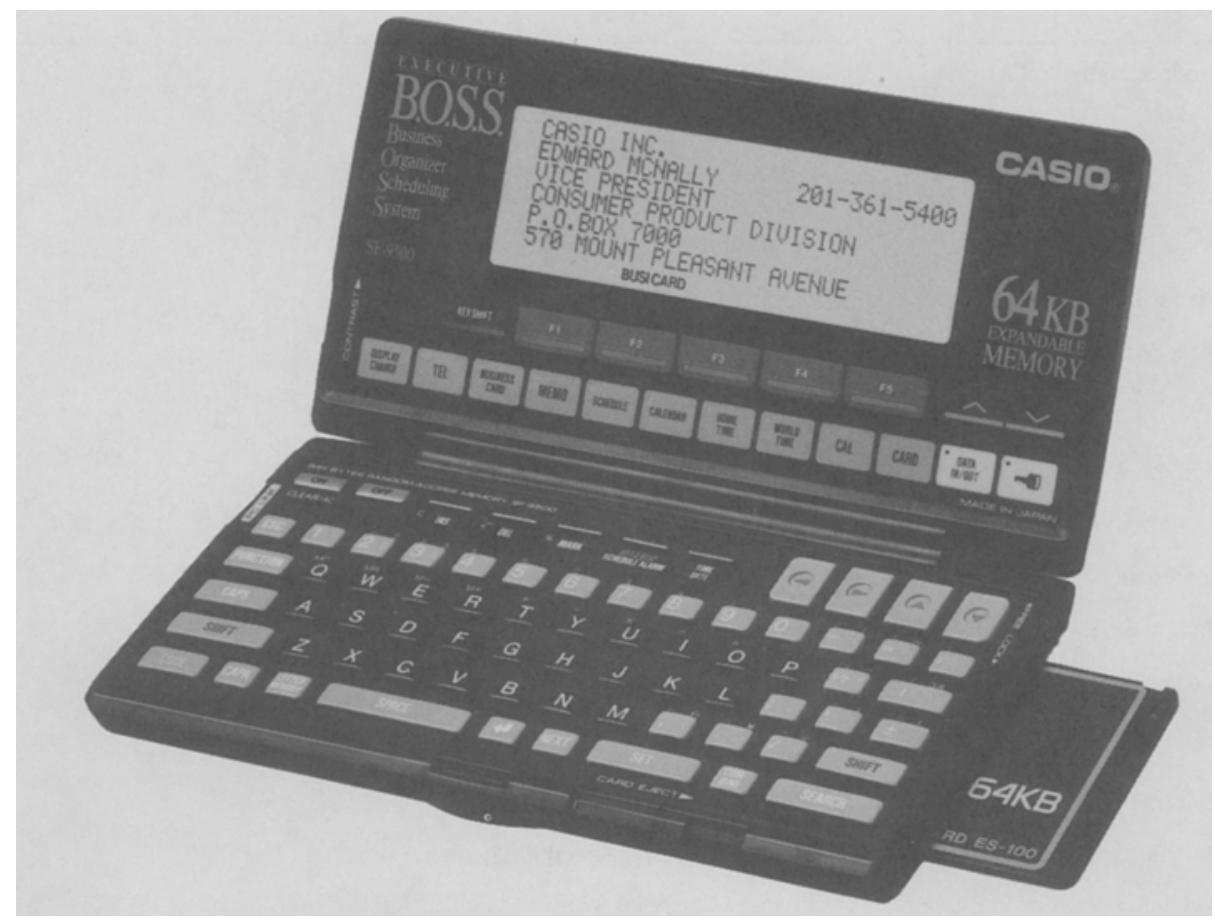

Figure 2. The Casio BOSS (Business Organizer Scheduling System) SF-9500 is Casio's most advanced product in production. It features up to $128 \mathrm{~K}$ of memory and insertion RAM cards for custom programs. In a split package design, the display is 32 characters by 6 lines, and the keyboard is typewriter style with several additional function buttons on both sides of the case. The display has a tilt-and-lock feature which makes the unit a miniature desktop terminal.

special medical VPC package, it is really too early to talk about vertical market standards in the industry, since we don't have a real product yet or an established customer base.

With the thrust of Selectronics into the electronic book area, there is a real question as to whether the other players in this field will see this as a significant market and offer a competitive package that can handle 8-16 megabytes of textual data.

Without this mass storage capacity, our efforts at reaching the physician will be very limited. A physician's memory is not really challenged until we approach some of the textbook sized databases that are considered references in the industry.

If we assume the space is available for storage of databases, we suddenly confront the question of convenience and packaging. Multiple cards or plastic modules will be needed with most of the units. If each application will require a separate card, this means that the user will be required to store the cards in the case of the VPC or carry them in his pocket. This only increases the bulk, weight, and complexity of the basic unit.

I don't believe doctors will use a device that will require a multitude of cards and a sequential insertion of these cards for each patient. The best package will be a composite database consisting of a series of critical reference files that can be accessed from one card 
Table $\mathfrak{3}^{a}$

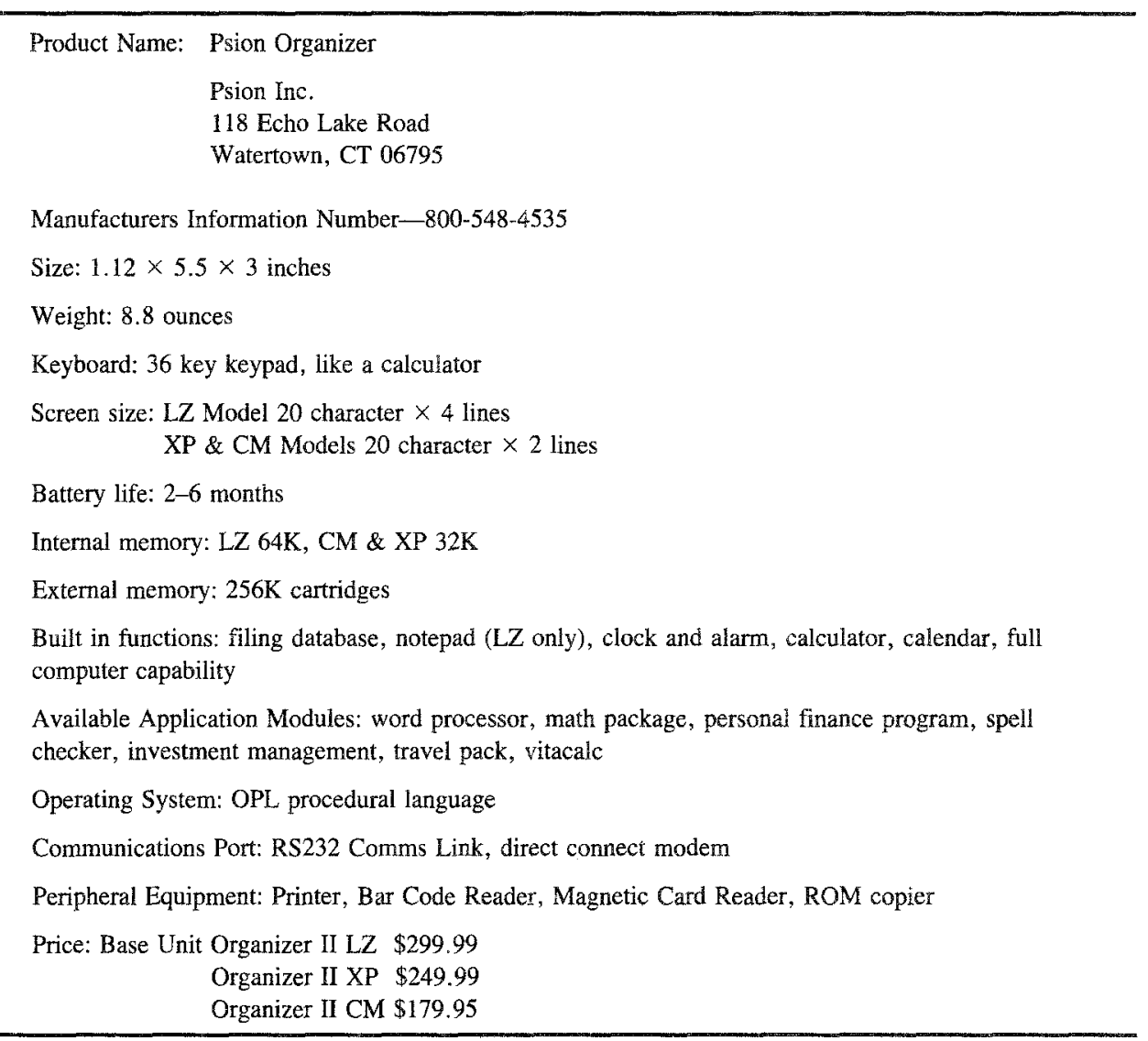

\footnotetext{
${ }^{a}$ See Figure 3.
}

loaded in the VPC. This parent database would contain data on drugs, diseases, and a host of practical subjects which will assist the physician in his daily work. The small handbooks that are available in the medical bookstore are a good basis for comparison and should provide a model of what is useful to the average physician as well as medical specialist.

If this composite database can be housed on one file in the VPC, then yearly updates can be made to this base unit so that there is no peripheral hardware or cards to deal with, but just one packaged unit that covers a variety of subjects.

To construct these composite databases, electronic publishers must screen the available publications and assemble their portfolio of material that will attract physician attention. This process will take several years and result in a consolidation of literature and publishing activity. Those who are successful in this area will have the best opportunity to establish their product in the coming years. The combination of good data and a low priced high performance platform will provide an irresistible temptation to the medical community. 


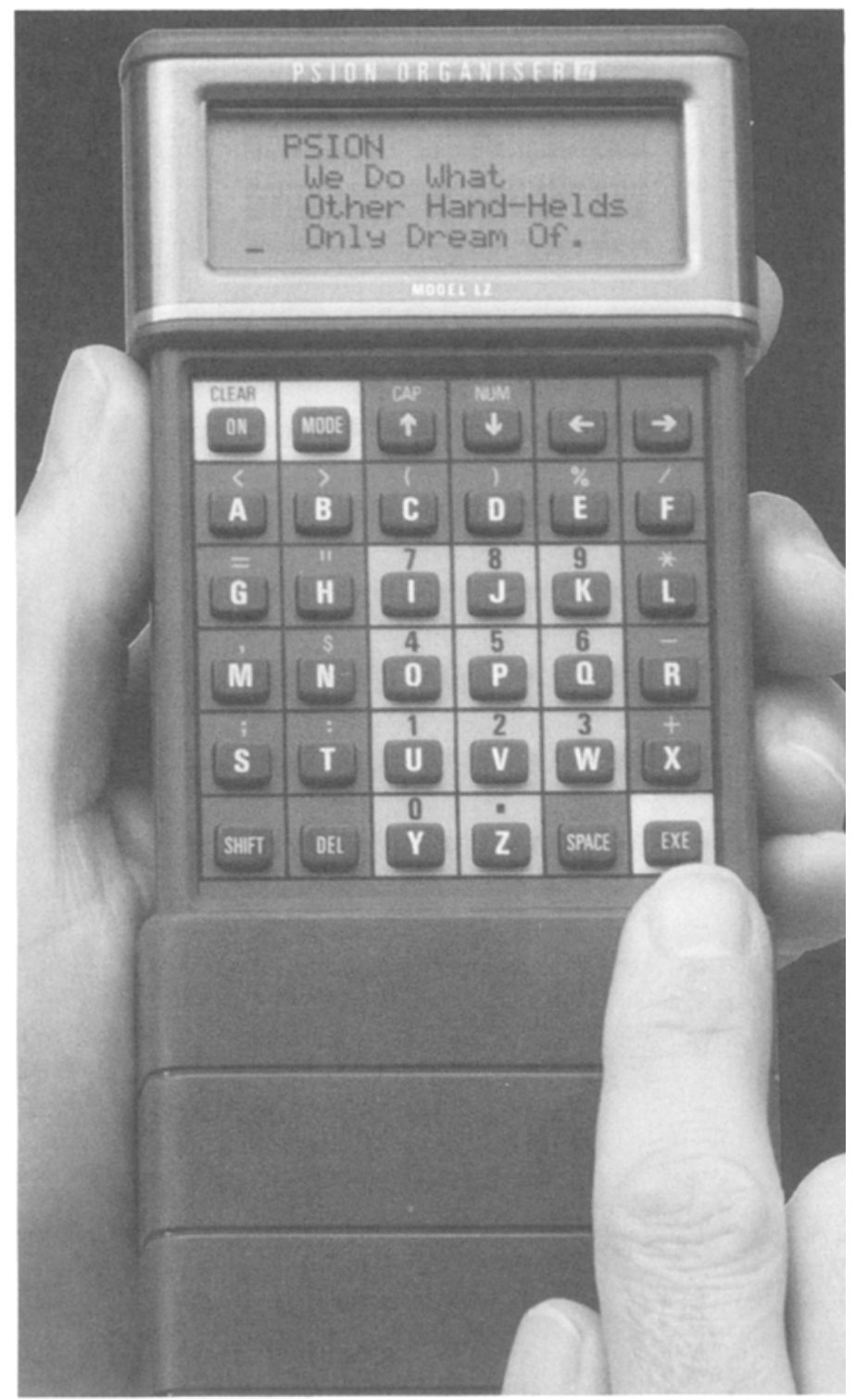

Figure 3. The PSION VPC is designed with a traditional calculator face. The case slides apart and exposes a matrix of alphanumeric keys that control data input. The screen is 20 characters by 4 lines and has a brightness control on the side of the screen. Memory packs can be added to the back of the package boosting available memory to $320 \mathrm{~K}$.

\section{FUTURE APPLICATIONS OF THE VPC}

\section{What Does the Future Hold for the Vest Pocket Computer?}

While it is still early, there are some obvious targets for immediate discussion:

1. VPCs will make it possible to package medical knowledge in a convenient form for low cost distribution. 
Table $4^{a}$

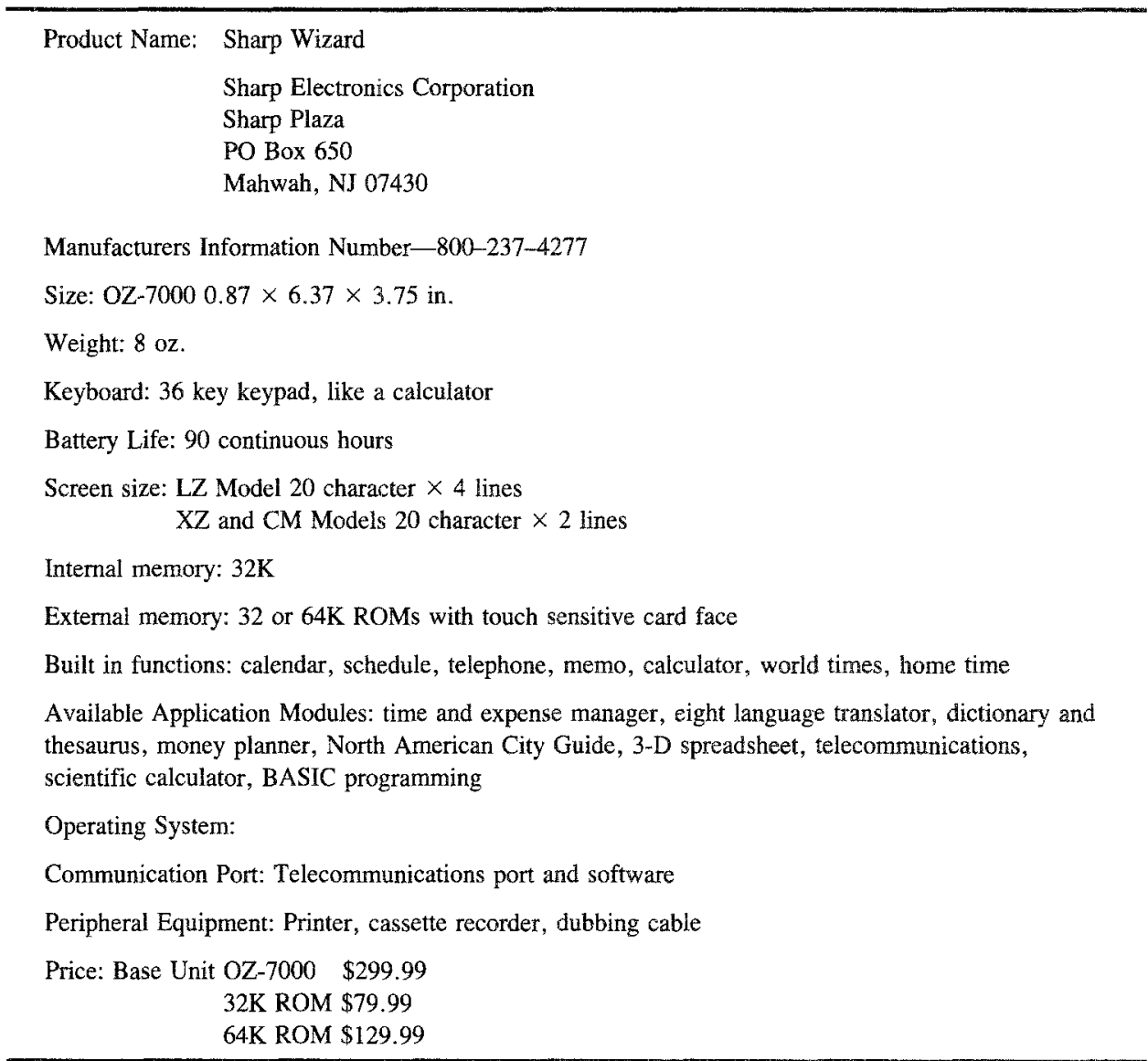

${ }^{a}$ See Figure 4.

A. The government and healthcare agencies may find this media most attractive for general announcements that will affect medical care. The government has many specialized databases that it could distribute it there was a low cost media of communication to those who could use them in their practice.

B. Hospitals have many unique databases that doctors are expected to know. The hospital formulary and laboratory normal values are only a few of the many internal files that are hospital specific. General knowledge of these basic parameters could form a new base for quality assurance and provide a level playing field for all healthcare workers. Nurses could have the same data available to doctors, and could cross-check hospital specific medications and treatments as needed.

C. Another significant use of this technology would be a paperless communication system for hospital staff and employees. Considerable money is expended duplicating hospital notes and memos. This could all be done electronically through the VPC.

D. At the physician level, memorization should become less of a factor in health- 


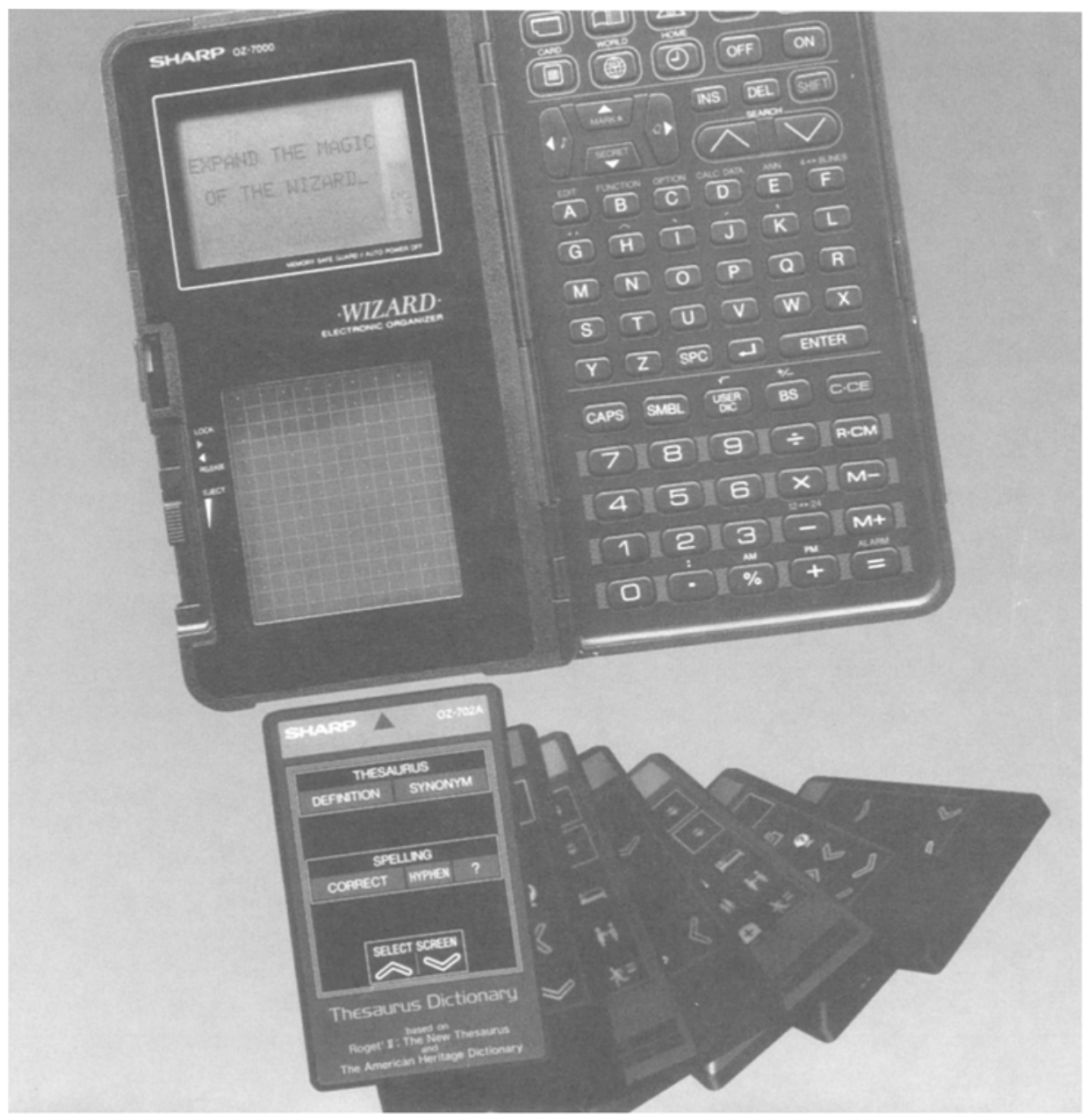

Figure 4. The Sharp Wizard is a compact split case computer with an LCD screen that shows 16 characters on 8 lines. The unit has an innovative memory card slot that offers touch sensitive pads on the face of the card for program control. With its vertical split design, the keyboard is calculator style with special function buttons for internal program control.

care delivery as more quality databases become available. These files include drugs, diseases, treatments, care plans, etc. All these subjects must be instantly accessible on the VPC at any site, and at any time. As the VPC storage capacity increases, one could use a desk top PC to host and compress larger specialized databases for pocket computer application.

E. Medical education can use the VPC concept very effectively by employing it in the basic teaching courses it offers. By creating ROM teaching modules, the instructors will be able to customize their courses for student use. Special 
Table $5^{a}$

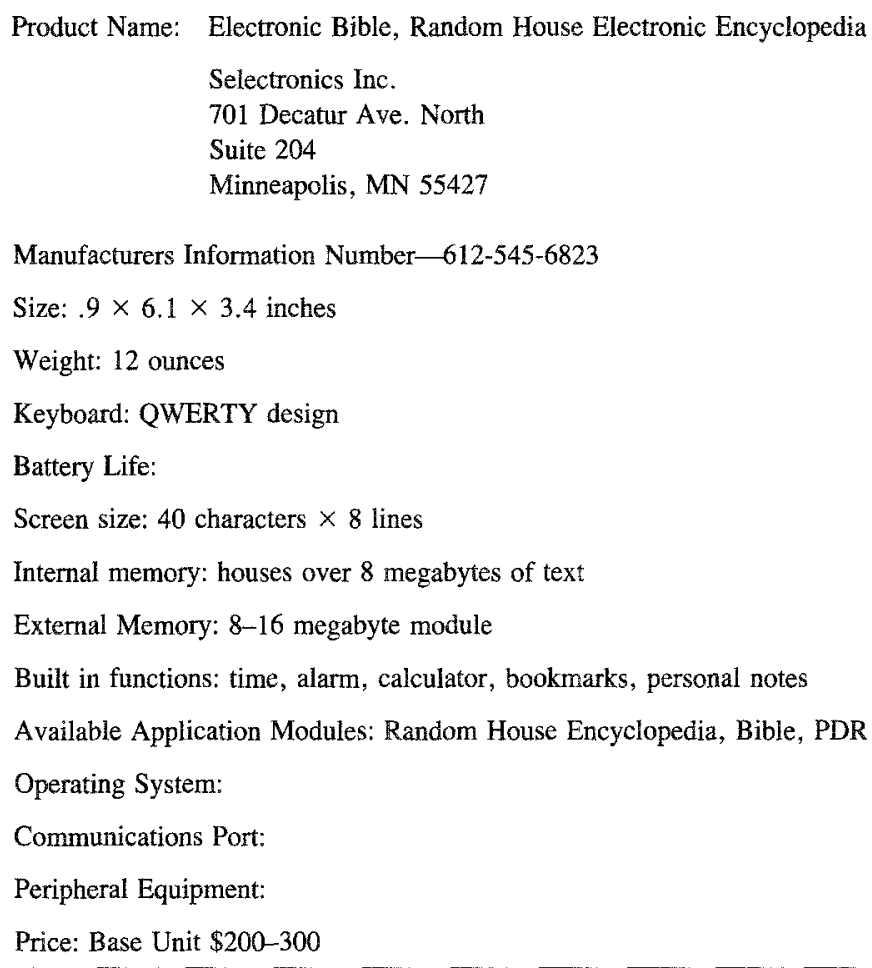

${ }^{a}$ See Figure 5 .

databases can be made, and problems and teaching materials associated into one coherent media. This technology will diminish the need for memorization and increase the expertise in the area of database usage and manipulation. These are skills that will improve the quality of general medical care in the future and make it possible to accelerate the learning process.

2. VPCs of the future are on the drawing board and will have obvious strengths and weaknesses.

A. The units will become smaller and lighter weight.

B. Larger screens and color displays will become standard equipment.

C. More internal processing power as well as more internal general business functions will emerge.

D. Growth in storage capacity is an absolute necessity. This should extend into the megabytes within the year for most manufacturers.

E. Direct communication to a PC, office, or hospital system will be routine.

F. Voice interaction may be possible to eliminate the need for a keyboard.

G. PC programs will be executed on the VPC with limited degradation.

H. As volume builds and the customer base increases, the prices will fall and more advanced units will be released into the market. 


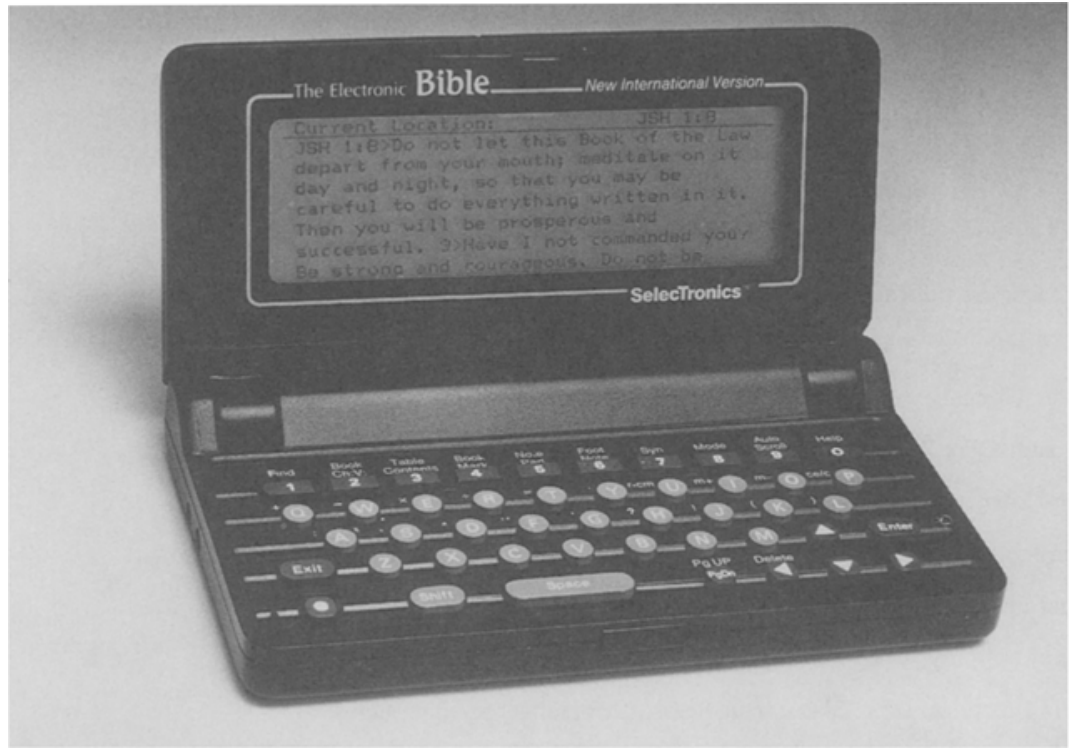

Figure 5. A complete Bible is contained in a small plastic case with attached keyboard. The unit offers special function keys as well as a QWERTY keyboard. Page controls are located in the lower right portion of the keyboard. A similar package exists for the Randon House Encyclopedia which contains over 8 megabytes of text.

I. A breakthrough will be needed for mass storage. One company is now demonstrating a small ROM disk that can store and recall hundreds of megabytes of data. Another alternative would be inexpensive credit cards with magnetic storage that could greatly expand the range of utility of the VPC and provide an inexpensive media for update of files and programs.

J. A marriage between the pager and the VPC is a natural upgrade.

$\mathrm{K}$. If we were to add a cellular phone to the package, you now have a space age tool for modern practice.

Can all this technology fit in one vest pocket? Individually, the VPC, pager and cellular phone are all vest pocket size. Whether this can actually be designed into one small pocket unit is unknown. If the market develops for the VPC, I am sure someone will see the logic and economic potential of integrating all these modes of communication, and will actually produce such an advanced system.

Until then-Until we can establish the VPC market for medical practice, all these advanced features will be nothing more than fanciful speculation. The work must be done now on the databases to build a foundation of quality software so that there will be a satisfied customer base on which to expand. Only the attraction of volume sales will support advanced hardware technology development and the commercial production of a device we would all like to own.

\section{REFERENCES}

1. 1990 Joint Report by the AMA Council on Scientific Affairs. Medical informatics: An emerging medical discipline, American Medical Association, Chicago, III., 1990.

2. Grams, R.R., Medical Information Systems-The Laboratory Module, Humana Press, 1979. 